\title{
Adolescente con autolesiones no suicidas en un entorno de adversidad psicosocial
} Adolescent with non-suicidal self-harm in a psychosocial adversity environment

\author{
Dr. Pablo del Brío Ibáñeza , Dra. Prof. Marta E. Vázquez Fernández ${ }^{b}$ y Dr. Prof. Carlos Imaz Ronceroc
}

\section{RESUMEN}

Las conductas lesivas no suicidas suponen un problema sanitario de creciente importancia, con una prevalencia en muestras comunitarias de adolescentes del 15-20\%. Las autolesiones pueden tener un significado diverso; se clasifican en intrapersonales o interpersonales; son un factor de riesgo y evolutivo, con inicio en la adolescencia temprana.

Se presenta el caso de una adolescente de 12 años con factores de riesgo psicosociales, que acudió a la consulta por cortes autoinfligidos en los brazos y las piernas, realizados con una cuchilla de afeitar, detectados en el colegio. Se intervino potenciando la autoestima y en regulación de emociones con intervención multidisciplinar (sistema educativo, servicios sociales, psiquiatría infantojuvenil y pediatría), con una evolución positiva.

Es vital su detección y abordaje con el paciente y su familia para evitar riesgos futuros, especialmente, de patología psíquica. La intervención se realiza desde la Atención Primaria, pero precisa la colaboración de otros profesionales.

Palabras clave: conducta autodestructiva no suicida, psiquiatría del adolescente, psiquiatría infantil.

\section{ABSTRACT}

Non-suicidal harmful behaviors pose a health problem of increasing importance, with a prevalence in community samples of adolescents of $15-20 \%$. Self-harm can have adverse meaning, qualifying in intrapersonal or interpersonal; they are a risk and evolutionary factor, with an age of onset in early adolescence.

We present the case of a 12-year-old adolescent with psychosocial risk factors, who went to the consultation for self-cuts in arms and legs, made with a razor, detected in the school. It was enhanced her self-steem an guided in regulation of emotions with multidisciplinary intervention (educational system, social services, infantile-juvenile Psychiatry and Pediatrics), with a positive evolution.

It is vital to detect and approach this issue with the patient and the family to avoid future risks, especially psychic pathology. The intervention is done from Primary Care, but it is necessary the collaboration of other professionals.

Key words: self-injurious behavior, adolescent psychiatry, child psychiatry.

a. Medicina de Familia, Centro de Salud Arturo Eyries.

b. Centro de Salud Arturo Eyries.

c. Unidad de Salud Mental Infantojuvenil.

Valladolid Oeste, España.

Correspondencia:

Dr. Pablo del Brío Ibáñez: pablodelbrio@gmail.com

Financiamiento: Ninguno.

Conflicto de intereses: Ninguno que declarar.

Recibido: 3-6-2018

Aceptado: 25-2-2019 http: / / dx.doi.org/10.5546/ aap.2019.e485

Cómo citar: del Brío Ibáñez P,Vázquez Fernández ME, Imaz Roncero C. Adolescente con autolesiones no suicidas en un entorno de adversidad psicosocial. Arch Argent Pediatr 2019;117(5):e485-e488.

\section{INTRODUCCIÓN}

La creciente incidencia de conductas autolesivas no suicidas en adolescentes es un problema de salud pública por el elevado coste social. ${ }^{1}$ La adolescencia es una etapa de cambios que puede aumentar el nivel de estrés y ocasionar un desajuste psicológico. ${ }^{2}$ La relación que establecen con su entorno, la vulnerabilidad que, generalmente, acompaña este tipo de conductas, asociada a los cambios biológicos inherentes a la edad juvenil son el estrato que puede causar la adopción de conductas de riesgo como respuesta patológica a la falta de regulación emocional. ${ }^{3}$ Dada la importancia clínica, evolutiva y de pronóstico de los rasgos y trastornos de personalidad en los pacientes con autolesiones, se decidió presentar este caso clínico.

\section{CASO CLÍNICO}

Se trata de una adolescente de 12 años, que acudió a la consulta de Pediatría acompañada de su padre porque, en el colegio, habían descubierto cortes autoinfligidos en los brazos y en las piernas. Procedía de un embarazo no deseado, sin problemas durante la gestación ni el período perinatal. Era la sexta de seis hermanos. Se trataba de una familia inmigrante procedente de Argentina; la paciente había llegado a España a los 2 años. Habían realizado escasas consultas y revisiones en nuestro centro de salud, pero había completado el calendario vacunal.

En la exploración, se descubrieron lesiones en los brazos y en las piernas que se realizaba ella misma con una cuchilla de afeitar. Eran cortes poco profundos, que buscaban figuras geométricas, en diferente estado de evolución (Figura 1 y 2). Además, presentaba desarrollo puberal completo. 
La joven y los padres se entrevistaron por separado. La madre no refirió problemas con sus hijos, pero reconoció haber sido excesivamente protectora con su hija más pequeña. El padre refirió haberse visto afectado por el suicidio de su padre cuando tenía 6-7 años y haber estado separado del núcleo familiar durante 10 años por motivos laborales (en España, mientras su familia estaba en Argentina). Aunque ambos padres trabajaban, tenían ingresos bajos y, recientemente, habían necesitado cambiar su domicilio por la crisis económica a otro de menor coste y más pequeño.

La adolescente refirió haber visto este tipo de prácticas en Internet y no sabía bien por qué se las hacía, no era un intento autolítico. Cuando estaba nerviosa, le servían de desahogo y la relajaban. Refirió que le costaba relacionarse con las compañeras de clase. El color oscuro de su piel la hacía sentirse diferente; le había provocado cierto rechazo y bullying de sus compañeras. Veía a su madre como una progenitora dominante, impulsiva y con carácter. Negó el consumo de drogas o medicamentos y alcohol; solo reconoció haber probado el tabaco. No tenía problemas alimentarios.

Ante esta situación, se inició un tratamiento con abordaje multidisciplinar en el que participaron los Servicios Sociales, que los incluyeron en un programa de apoyo familiar
FIGURA 1. Lesiones en el antebrazo

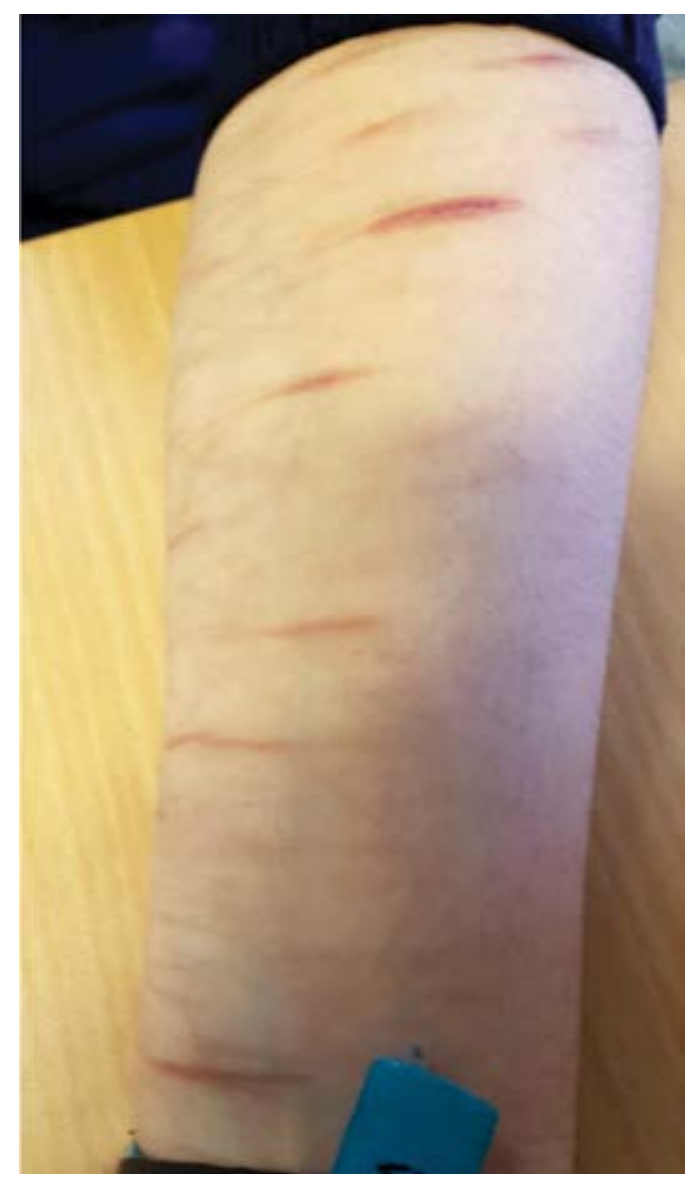

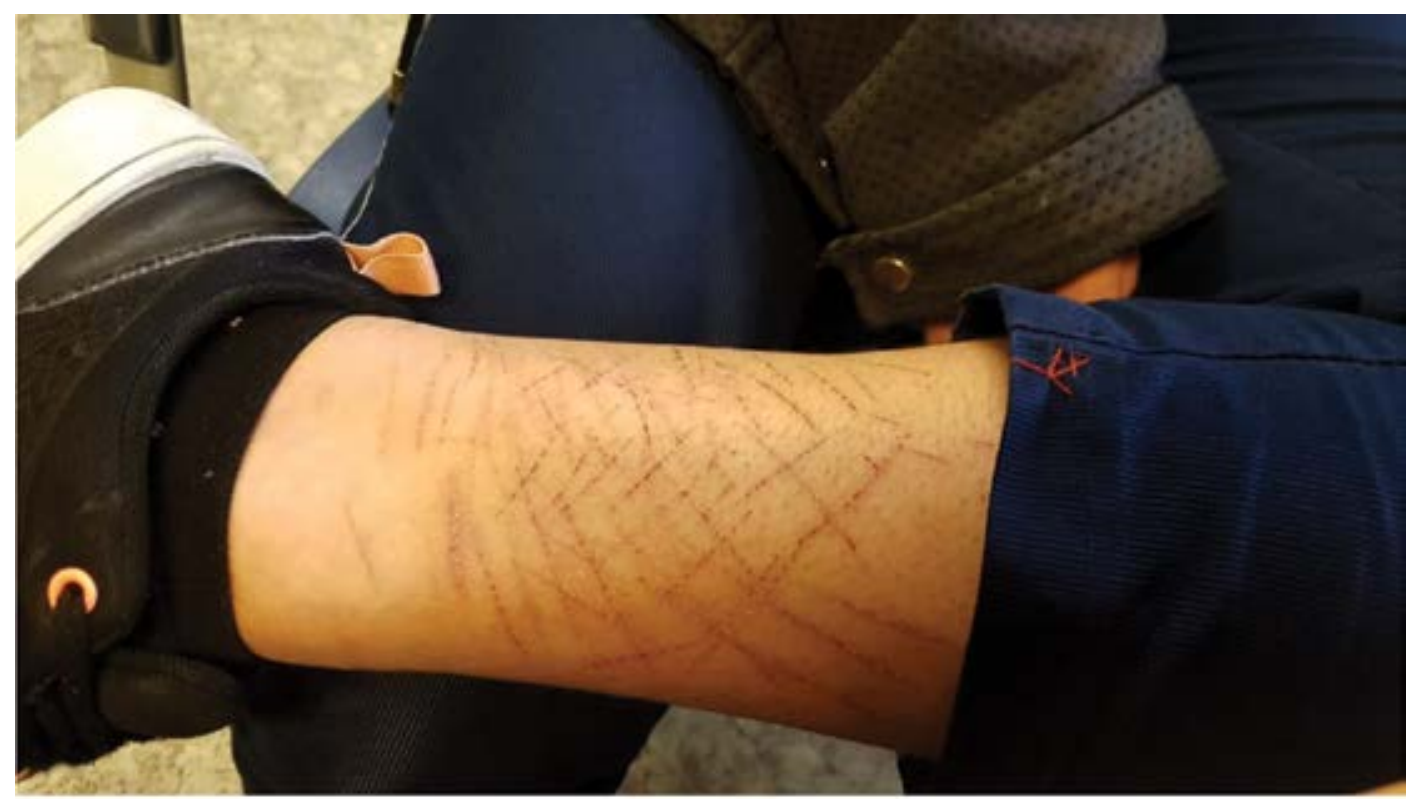

Se puede ver la diferencia respecto a las del antebrazo, que tienen mayor tiempo de evolución. 
(para superar las dificultades de relación, organizativas y económicas), Sistema Educativo para resolver los problemas individuales e intrapersonales y evitar su cronificación, la Unidad de Psiquiatría Infantojuvenil y el equipo de Atención Primaria pediátrica, en el que se trabajaba el manejo inadecuado de las emociones y la baja autoestima.

La evolución fue satisfactoria, mejoró su rendimiento escolar y estaba más integrada, aunque continuaba realizándose algún corte esporádico. Se descubrieron más casos de autolesiones en compañeras de clase, que estaban siendo tratadas.

\section{DISCUSIÓN}

Las autolesiones son conductas deliberadas destinadas a producir a uno mismo daño físico leve o moderado. La bibliografía demuestra que son más frecuentes en los adolescentes y en los adultos jóvenes, con tasas del $15 \%$ al $20 \%$, independientemente de la localización geográfica. En los adultos, solo llegan al $6 \%$. El inicio suele ser a los 13 años, sin diferencia en cuanto al género. Parece haber una diferencia entre los hombres y las mujeres respecto a los métodos utilizados para la realización de las lesiones: las mujeres usan más el corte como medio de autolesión; los hombres suelen golpearse o quemarse. ${ }^{4}$ En general, las cifras son más altas con antecedentes o patología psiquiátrica. ${ }^{5}$

El uso de diferentes metodologías en la evaluación (muestreo, cuestionarios o escalas y marco de tiempo) y de distintos sistemas de clasificación para las autolesiones son las principales limitaciones a las que hay que enfrentarse para la interpretación de los estudios epidemiológicos. Además, se utilizan varios términos para definir la autolesión. En Europa y Australia, "autolesión deliberada" define conductas autolesivas con y sin intención suicida, pero sin resultados fatales. Los estudios publicados en Canadá y en Estados Unidos emplean "autolesión no suicida", que excluye explícitamente la ideación suicida. ${ }^{6}$

La forma de autolesionarse es variada. La más habitual es la producción de cortes. Las zonas elegidas son, principalmente, las muñecas y los brazos; otras son más infrecuentes. ${ }^{7}$ Se utilizan tijeras, cuchillas de afeitar, fragmentos de vidrio, metal $u$ otros objetos punzantes. Otras formas son la inserción de objetos bajo la piel, el rascado excesivo, morderse la piel periungueal, pellizcarse, quemarse o golpearse. ${ }^{8}$
La autolesión se interpreta como una respuesta patológica a la falta de regulación emocional y tolerancia a las agresiones externas, en la que se busca un alivio rápido de emociones no deseadas. Un elevado porcentaje de adolescentes afirma que el dolor es escaso o inexistente en comparación con su sufrimiento emocional, y la autolesión resulta en la reducción de emociones negativas, así como en la producción de sentimientos de calma y alivio. Algo más de la mitad de las personas que se han autolesionado lo reconocen como una forma de enfado autodirigido o autocastigo; así, se puede establecer una relación causal entre la autocrítica y las conductas autolesivas, motivo por el que suelen repetirse. También pueden aparecer con el objetivo de influir en otros o producir un signo físico de angustia y verse en adolescentes "normales" por diversos motivos: llamada de atención, imitación, hacerse famoso en Internet y otros.,

Las autolesiones no suicidas se pueden dividir en dos categorías: intrapersonales o autocentradas, para la regulación de las emociones y el autocastigo, e interpersonales, enfocadas en el otro, principalmente, dirigidas a influenciar. ${ }^{9}$ El uso de Internet relacionado con el suicidio y las autolesiones es común entre los adultos jóvenes, en especial, entre aquellos con pensamientos y conductas suicidas. ${ }^{10}$

Existen factores asociados: riesgo psicosocial, inestabilidad emocional, síntomas depresivos y ansiosos, consumo de sustancias, trastorno de conducta alimentaria, trastorno de déficit de atención e hiperactividad, violencia familiar, abuso sexual y otras conductas autolíticas. ${ }^{11}$

En el caso que se presenta, la adolescente se encuentra en una situación de vulnerabilidad que ayuda o facilita el inicio de las conductas autolesivas. Tanto los factores internos (baja autoestima por verse diferente) como externos (exclusión social, bajo nivel económico y el uso poco adecuado de las redes sociales) pueden haber influido.

Las conductas autolesivas y las suicidas difieren en puntos importantes. La frecuencia es muy superior en las conductas autolesivas, y los métodos son distintos: en los suicidas, las lesiones ocasionadas son más graves y letales. El objetivo final tampoco es el mismo. Pero estas diferencias no impiden su coexistencia. Algunos estudios muestran que existe relación epidemiológica entre ambas, ya que, tras una autolesión, el riesgo de suicidio oscila entre el $0,5 \%$ y el $2 \%$ al año siguiente. ${ }^{12} \mathrm{El}$ riesgo relativo de autolesionarse 
en el grupo de menores con algún pensamiento suicida es, aproximadamente, 10 veces mayor que en el grupo que no ha tenido estos pensamientos antes. ${ }^{13,14}$

Para estudiar y guiar las intervenciones clínicas, se utilizan cuestionarios; la Escala de Motivación de la Autolesión (Self-Injury Motivation Scale, SIMS) cuantifica el motivo y la frecuencia de las autolesiones, evalúa el control de emociones y el objetivo. La Escala de Evaluación Funcional de la Automutilación (Functional Assessment of Self-Mutilation, FASM) examina los métodos de autolesión y su frecuencia. También la entrevista estructurada de intento de suicidio por lesión autoinfligida (Suicide Attempt SelfInjury Interview, SASII) examina los métodos de autolesión, la intención o motivación, la frecuencia y la letalidad. ${ }^{15}$

Su detección es vital para una intervención precoz e implementación de medidas preventivas por la familia, los educadores y los agentes de salud. Si no existe un cribado, se corre el riesgo de que estas conductas se cronifiquen y las comorbilidades psiquiátricas no reciban el tratamiento necesario. El objetivo es orientar al núcleo familiar sobre el uso responsable de redes sociales e Internet, y hacer hincapié en la necesidad de supervisión y acompañamiento por parte de los adultos, sobre todo, en edades tempranas. Además, se fomentará el desarrollo de estilos de comunicación saludable con sus hijos adolescentes.

En condiciones ideales, el abordaje deberá ser interdisciplinar y dependerá de los recursos con que se disponga. En casos leves o que tienen el antecedente de autoagresiones alejado en el tiempo, bastará con la orientación al paciente y a su familia, y los casos más complejos requerirán seguimiento clínico, tratamiento psicológico y apoyo de otras especialidades.

Como conclusión, se debe destacar que, debido al aumento de la prevalencia de autolesiones en la adolescencia, es importante su detección y evaluación de manera rutinaria durante la atención pediátrica. Si bien pueden presentarse en cualquier adolescente, son más frecuentes asociadas a factores de riesgo psicosocial.

\section{REFERENCIAS}

1. Nock MK. Self-injury. Annu Rev Clin Psychol. 2010; 6(1):339-63.

2. CostelloEJ, MustilloS, Erkanli A, Keeler G, et al. Prevalence and development of psychiatric disorders in childhood and adolescence. Arch Gen Psychiatry. 2003; 60(8):837-44.

3. Najman JM, Heron MA, Hayatbakhsh MR, Dingle K, et al. Screening in early childhood for risk of later mental health problems: A longitudinal study. J Psychiat Res. 2008; 42(8):694-700.

4. Klonsky ED, MuehlenkampJJ.Self-injury: a research review for the practitioner. J Clin Psychol. 2007; 63(11):1045-56.

5. Klonsky ED, Victor SE, Saffer BY. Nonsuicidal self-injury: what we know, and what weneed to know. Can J Psychiatry. 2014; 59(11):565-8.

6. Muehlenkamp JJ, Claes L, Havertape L, Plener PL. International prevalence of adolescent non-suicidal selfinjury and deliberate self-harm. Child Adolesc Psychiatry Ment Health. 2012; 6:10.

7. Muñoz Muñoz A, Arroyave López J, Romero Montoya T. Generalidades en la Evaluación Clínica Psicológica. Referentes a las autolesiones no suicidas en adolescentes. Psyconex. 2016; 8(13):1-11.

8. Nixon MK, Cloutier P, Jansson SM. Nonsuicidal selfharm in youth: a population-based survey. CMAJ. 2008; 178(3):306-12.

9. Klonsky ED, Glenn CR. Assessing the functions of non-suicidal self-injury: psychometric properties of the Inventory of Statements About Self-injury (ISAS). J Psychopathol Behav Assess. 2009; 31(3):215-9.

10. Mars B, Heron J, Biddle L, Donovan JL, et al. Exposure to, and searching for, information about suicide and self-harm on the Internet: Prevalence and predictors in a population based cohort of young adults. J Affect Disord. 2015; 185:239-45.

11. Kirchner T, Ferrer L, Forns M, Zanini, D. Conducta autolesiva e ideación suicida en estudiantes de enseñanza secundaria obligatoria. Diferencias de género y relación con estrategias de afrontamiento. Actas Esp Psiquiatr. 2011; 39(4):226-35.

12. Gómez Sánchez S, Sevillano Benito I, Geijo Uribe S, Mongil López B, et al. Autolesiones en una Unidad de hospitalización Psiquiátrica infanto-juvenil. Psiquiatr Biol. 2018; 25(1):2-6.

13. Wilkinson PO. Nonsuicidal self-injury: a clear marker for suicide risk. J Am Acad Child Adolesc Psychiatry. 2011; 50(8):741-3.

14. Muehlenkamp JJ, Gutierrez PM. An Investigation of Differences between Self-Injurious Behavior and Suicide Attempts in a Sample of Adolescents. Suicide Life Threat Behav. 2004; 34(1):12- 23.

15. Nock MK, Prinstein MJ. A Functional approach to the assessment of Self-mutilative behavior. J Consult Clin Psychol. 2004; 72(5):885-90. 